\title{
LA CIUDAD COMO ESCENARIO: LOS HABITANTES DE LA CALLE Y SUS EXPERIENCIAS URBANAS
}

THE CITY AS SCENE: THE PEOPLE OF THE STREET AND URBAN EXPERIENCES

\author{
Paula Cecilia Rosa ${ }^{1}$
}

\section{Resumen}

El artículo se centra en la problemática de los habitantes de la calle, como son definidos en el marco de la investigación realizada. Es decir, varones, mujeres o grupos familiares que transcurren su cotidianeidad en las plazas, veredas y calles de la ciudad. Las prácticas y recorridos de los habitantes de la calle en la ciudad redefinen las concepciones de espacio público generando cambios en sus usos habituales. Su modo de vida genera ciertos efectos dado que corrompen con su presencia una "regla" de uso y convivencia en lo público: "allí nadie habita" pues es un lugar de circulación, anonimato y de relaciones sociales precarias. En este sentido, es que presentaremos las experiencias urbanas cotidianas de esta población centrándonos en los recorridos que realizan por la ciudad como así también en los vínculos sociales que entablan. Entendemos que la experiencia urbana de los habitantes de la calle permite realizar un acercamiento diferente a los estudios de lo urbano pues resulta un caso interesante para acercarse a los modos posibles de habitar la ciudad.

Palabras claves: habitantes de la calle-ciudad-relaciones sociales

\section{Abstract}

The article focuses on the problem of inhabitants of the street, as they are defined in the framework of the investigation. It means, men, women or family groups that run their daily life in the streets, sidewalks and city streets. The practices and courses of the inhabitants of the street in the city redefine the conceptions of public space leading to changes in their typical uses. Their way of life generates certain effects because with their presence they corrupt a "rule" of use and coexistence in public: "no one lives there" because it is a place of movement, anonymity and poor social relationships. In this regard, we will present the everyday urban experiences of this

\footnotetext{
${ }^{1}$ Doctora en Ciencias Sociales - Universidad Nacional General Sarmiento. Investigadora Asistente Carrera de Investigador Científico y Tecnológico del Consejo Nacional de Investigaciones Científicas y Técnicas (CONICET). Docente de Metodología de Investigación Social, Universidad de Buenos Aires - UBA. Miembro del Programa Integral de Economía Solidaria de la Universidad Nacional de San Martín - UNSAM. E-mail: paula_rosa00@yahoo.com.ar
}

Revista de Direito da Cidade, vol.07, no 02. ISSN 2317-7721 pp.518-536 
population focusing on the journeys made by the city as well as in the social bonds that engage. We understand that the urban experience of the inhabitants of the street allows for different urban studies because it is an interesting case to approach to possible ways of inhabiting the city.

Keywords: inhabitants of the street, city, social relationship

\section{INTRODUCCIÓN}

El espacio es un lugar practicado. De esta forma, la calle geométricamente definida por el urbanismo se transforma en espacio producido por la intervención de los caminantes. (De Certeau, 2000:129)

Este artículo analizará la ciudad apartándose de los análisis que la entienden desde una concepción material solamente para ubicarse dentro del campo de análisis de las relaciones sociales desarrolladas en el ámbito urbano. Siguiendo a Lindón (2009) podemos afirmar que los estudios sobre la espacialidad y la ciudad se han centrado en el análisis de los espacios residenciales, habitacionales, de consumo o bien en los medios de transporte o la movilidad espacial dentro de la ciudad pero poco se trabaja sobre el habitante de la ciudad. Es inusual el análisis sobre los recorridos que realiza en su cotidianeidad, los lugares a los cuales asiste, el uso que hace de los espacios públicos, cuáles son sus lugares de ocio, de trabajo, qué vínculos entabla, etc. En este sentido, es que la experiencia de los habitantes de la calle, es decir, varones, mujeres o grupos familiares que transcurren su cotidianeidad en las plazas, veredas y calles de la ciudad, permite realizar un acercamiento diferente a los estudios de lo urbano pues resulta un caso interesante para acercarse a los modos posibles de habitar la ciudad. De este modo, entendemos siguiendo a Rizo (2013) que:

no debemos abordar el espacio urbano sólo como la dimensión física de la ciudad, sino que es fundamental incorporar la experiencia de quienes habitan en ella. Y esta idea se complementa con que las experiencias de vivir en una ciudad son muy diversas y dependen de las expectativas, los logros, las frustraciones, etc., de los sujetos (Rizo, 2013:73).

Así, siguiendo a Lindón (2009), sostenemos que al concebir al sujeto espacialmente se reconoce que su actuar en el mundo hace y modela los lugares y, al mismo tiempo, que los lugares que habita le dejan marcas.

Revista de Direito da Cidade, vol.07, no 02. ISSN 2317-7721 pp.518-536 
El artículo tiene como objetivo ahondar en la problemática de los habitantes de la calle partiendo de la premisa de que al habitar el espacio público son también habitantes de la ciudad aunque su modo de vida se separe de lo esperado para este ámbito. En este sentido, se presentará la definición de habitantes de la calle categoría analítica construida para dar cuenta de la vinculación entre las personas que habitan en el espacio público y su relación con el entorno en el cual habitan. Para ello, se describirán los recorridos, vivencias y relaciones que esta población tiene en la ciudad. En definitiva, partimos de la idea de que la experiencia urbana que poseen los habitantes de la calle permite poner en cuestión ciertas caracterizaciones que se realizan del habitante de la ciudad y de la ciudad misma como un lugar de circulación, de anonimato y de lo efímero.

Las reflexiones realizadas en este artículo surgen en el marco de la tesis doctoral "Entramado de relaciones: Organizaciones de la Sociedad Civil y la asistencia a los habitantes de la calle en la Ciudad Autónoma de Buenos Aires" (2012), elaborada a partir de una investigación cualitativa de cinco años de duración (2007-2010) que implicó un involucramiento en la problemática de los definidos como habitantes de la calle. Para el desarrollo de la tesis doctoral se hicieron entrevistas semi-estructuradas a habitantes de la calle, miembros de organizaciones sociales y funcionarios estatales de la ciudad de Buenos Aires junto a la realización de observaciones participantes en diversos ámbitos (espacios públicos, organizaciones sociales, instituciones estatales, etc.). De este modo, lo elaborado en el artículo se basa en fuentes primarias generadas por la propia autora durante su trabajo de campo y sus posteriores análisis.

El artículo se estructura del siguiente modo: Primero, se presenta brevemente el modo de vida del típico Habitante de la ciudad, el urbanita; luego en el apartado Habitantes de la calle se ahondará en esta categoría analítica construida en el marco de la investigación; a continuación, la sección La ciudad como oportunidad se centrará en las prácticas cotidianas que realizan los habitantes de la calle por la ciudad, aquí se dará a conocer sus recorridos y movimientos que efectúan trazando "un mapa específico de la metrópolis"; en el último apartado Construcción de vínculos se presentarán las particularidades del modo de vida de los habitantes de la calle en función de los vínculos sociales que entablan en su recorrido por la ciudad; finalmente, se presentan las Reflexiones finales. 


\section{HABITANTES DE LA CIUDAD}

Desde diversas tradiciones teóricas se entiende que el típico representante de la vida urbana moderna es el urbanita. Este habitante de la ciudad transcurre su vida en el anonimato, la individualización y la superficialidad. Este sujeto urbano se vincula con la ciudad desde el consumo, la circulación y/o el entretenimiento. De este modo, se entiende que desarrolla relaciones e intercambios sociales impersonales, transitorios y basados en intereses y contratos. Sus relaciones son generalmente fluctuantes, racionales y aleatorias. Este intercambio social es propio de la vida de las grandes ciudades generando hasta un tipo específico de forma psíquica (Simmel, 1986). E urbanita transita por la ciudad de un modo funcional a sus necesidades diarias como son concurrir al ámbito laboral, alimentarse, desarrollar ciertos tipos de entretenimientos que la vida urbana le ofrece, generar vínculos funcionales a sus necesidades, etc. En este sentido, Lofland (1998) (citada en Guillamón, 2008), señala que existen principios que regulan las interacciones en los espacios públicos urbanos. Entre ellos define la "movilidad cooperativa" que se "refiere al carácter coreográfico de la conducta de los peatones, al trabajo conjunto entre extraños para organizar los trayectos en el espacio y evitar choques" (Gillamón, 2008:81) y la "inatención cívica" que se vincula a la mirada en el espacio público a otro transeúnte. Esta se basa en mostrar al otro que se está atento a su presencia pero que no es objeto de una curiosidad o de una atención particular. Al hacer este gesto de cortesía visual, la mirada del primero puede cruzarse con la del otro sin por ello autorizarse un "reconocimiento". De este modo, se puede decir que en el espacio público se desarrollan relaciones:

Generadas a partir de códigos y sistemas de interacción pactados sobre la emergencia de las situaciones, a las que el individuo sobrevive gracias a los "saberes prácticos" aprendidos a lo largo de su devenir como usuario (De la Peña, 2013: 46).

Estas "formas de ser y de relacionarse" constituyen un modo de vida y son vistas como una cualidad compartida por los habitantes de la ciudad de todas partes del mundo pues "el ciudadano metropolitano en la esfera pública oscila entre la gentil indiferencia y la vehemente instrumentalidad" (Torterola, 2012:135). Estas prácticas establecen los modos legítimos de 
habitar, transitar o pertenecer a la ciudad y están socialmente aceptadas. Sin embargo, en las ciudades existen otras prácticas y sujetos que no gozan la misma legitimidad.

En el campo de los estudios urbanos, la Escuela de Chicago es considerada como la pionera en la indagación del modo de vida urbano. Durante las primeras décadas del siglo XX esta Escuela tuvo el mérito de inaugurar un nuevo campo de investigación que tenía a la metrópolis como centro del interés científico (Torterola, 2012). Siguiendo con esta línea es que se realizó el estudio de la subcultura del "hobo" a cargo del joven investigador Nels Anderson. "The Hobo" (1936) es una clásica obra sociológica de la "cultura marginal" que describe y analiza la vida de un tipo de sujeto urbano. Este sujeto es entendido como parte de una "cultura" específica que tiene sus formas de reproducción y de cambio, sus códigos, normas y lenguaje. Su particularidad radica en su inestabilidad laboral que lo llevada a una vida de movilidad (Viotti, 2008). Su particularidad radicaba en ser un itinerante que no tenía puesto de trabajo fijo ni residencia estable, por ello se movía sin seguir ningún plan. Este modo de vida lo constituía en un "desarraigado". Según De La Pena (2013):

El hobo, que comienza su carrera rompiendo los vínculos locales que le unen a su familia y a su vecindario, termina por romper todos los demás tipos de asociaciones. No sólo es un vagabundo, sino también un hombre sin razón de ser y sin patria. (De La Pena, 2013, citando a Park, cit. en Remy y Voyé, 1976: 225-226).

La figura del Hobo daba paso a la identificación de nuevas formas de organización social en el ámbito urbano pues evidenciaba la realidad de un tipo de sujeto que no lograba insertarse según los patrones establecidos socialmente. El Hobo expresa el arquetipo del individualismo pues es "un extranjero en su propio escenario urbano" (Torterola, 2012:135). Su modo de vida se vincula con la caracterización que se realiza también del urbanita pues comparte "algo de su movilidad y desarraigo" (De La Pena, 2013: 39). Sería entonces, junto al urbanita, un representante de los nuevos estilos de vida identificados en las grandes ciudades.

Esta caracterización de la vida urbana posee muchos puntos de contacto con la vida actual en las ciudades contemporáneas. La imagen del desarraigo, el individualismo, el anonimato, etc. continúan describiendo este modo de vida. Sin embargo, esta concepción invisibiliza a un tipo de sujeto que busca generar en su experiencia urbana la oportunidad de reproducir su vida y de 
constituir relaciones dadoras de sentido y pertenencia. Hacemos referencia a los habitantes de la calle que son personas que desarrollan su vida cotidiana en el espacio urbano.

\section{HABITANTES DE LA CALLE}

Teniendo en cuenta las múltiples definiciones existentes para referirse a esta población (personas en situación de calle, sin techo, moradores, etc.) y las cargas de sentido que cada uno de ellas posee ${ }^{2}$, se consideró necesario construir una nueva categoría analítica para definir a estas personas. Por ello se los comenzó a denominar en el marco de la investigación realizada como "habitantes de la calle". Esta definición es considerada más abarcativa y compleja dado que se entiende que en esta enunciación el énfasis está puesto en el medio en donde la persona habita, desarrolla su vida cotidiana y entabla vínculos sociales:

Se habla de habitantes porque se entiende que estos habitan el espacio de la calle pues entablan en ella una relación con el entorno y establecen vínculos e interacciones con diferentes personas y grupos que se encuentran en su misma situación como con otros que no (vecinos, comerciantes, transeúntes, etc.). (Rosa, 2012a y 2012b).

Con esta definición se buscó hacer hincapié en una cuestión poco contenida en las denominaciones más clásicas como es la utilización de los espacios públicos como ámbitos en los cuales desarrollar la vida cotidiana y entablar vínculos. De este modo, se busca entender la vida en la calle no solo como una condición física territorial específica de una situación social, sino como "[...] un contexto socio-cultural, un espacio de redes de relaciones que vehiculizan las interacciones sociales" (Marcús, 2006:102).

Para los habitantes de la calle el espacio urbano en el cual desarrollan su vida posee un significado particular. A partir de las prácticas realizadas y los sentidos otorgados a las mismas, los

\footnotetext{
2 "En la bibliografía sobre la temática, en artículos periodísticos y hasta en documentos de políticas públicas se las denomina como "personas en situación de calle" o "sin techo". La denominación "sin techo" prácticamente dejó de utilizarse para definir a esta población dado que se consideraba que caracteriza de un modo negativo a aquellos que son enunciados de este modo. La categoría hacía énfasis en sus carencias especialmente habitacionales- $y$, además, en muchos casos, se la empleaba como un descalificativo. En la actualidad el término más utilizado, tanto por el Estado como por las Organizaciones de la sociedad Civil, es el de "personas en situación de calle". Esta categoría fue propuesta desde el Estado y es empleada por las
} 
habitantes de la calle realizan un proceso de apropiación del espacio público en el que habitan. Lo significan y modifican, es por esto que se entiende el habitar como "[...] el proceso de significación, uso y apropiación del entorno que se realiza en el tiempo" (Signorelli, 2006 en Duhau y Giglia, 2008:22). Se hace referencia al concepto de apropiación dado que se lo entiende como:

el proceso mediante el cual los grupos sociales hacen suyo el espacio significándolo. Es decir, generando identificaciones particulares sobre un lugar específico. En este proceso no sólo se le otorga sentido al espacio, sino que se generan elementos que favorecen la identificación y la pertenencia. Es un ejercicio en donde se extiende la identidad hacia fuera, al tiempo que se interioriza el espacio y su significación (hacia adentro) (Portal, 2009: 64).

Al habitar la calle desarrollan en ella su vida cotidiana, es por ello que en este espacio realizan ciertas actividades que son consideradas como pertenecientes al mundo de lo doméstico: preparar los alimentos, comer, dormir, higienizarse, etc. (Da Matta, 1997). Estas son actividades que en este caso se llevan a cabo en un espacio que se vincula con lo público y compartido. Por esa razón el ámbito de la calle cobra un sentido particular para quien vive allí (Rosa, 2012a). Por estas experiencias la ciudad es vivida de un modo particular para esta población al punto de que en algunos casos luego de habitar años en la calle llegan a considerarla como su hogar y no quieren dejar de vivir allí. Es decir, que encuentran en este ámbito un lugar de pertenencia, un lugar para refugiarse, un lugar en el que pueden vivir ${ }^{3}$. Se podría decir, entonces, que aprendieron a vivir en un espacio con otras características, en un mundo en movimiento y que posee tiempos y sentidos diferentes ${ }^{4}$. Después de un tiempo en la calle saben cómo moverse y qué estrategias desplegar:

organizaciones. Estas la utilizan dado que hace referencia a una "situación", es decir, que a partir de esta categoría se entiende que vivir en la calle es una situación transitoria” (Rosa, 2012a:299).

${ }^{3}$ Esta situación se vincula con lo que Saucedo y Taracena (2011) describen como "arraigo" a la calle. Para los autores, esta para las poblaciones que allí habitan representa un "espacio de recepción abierto del cual se puede ser parte". De este modo, establecen la idea de que estas poblaciones pueden "echar raíces" en el espacio público y desarrollar un sentimiento de pertenencia a este lugar a partir de considerarlo como un ámbito "familiar".

${ }^{4}$ En su libro A casa \& a rua (2002) Roberto Da Matta sostiene que la calle es considerada como un universo regido por el engaño y el malandraje. Es un mundo en donde todos están en lucha, la calle es pensada como una selva, como naturaleza. Esto es así pues el dominio de la calle es semi-desconocido, está semi-controlado y poblado de personajes peligrosos. Se piensa que en la calle viven los malandras, los delincuentes, "así en la calle y en la mata es donde viven los malandros, los marginales y los espíritus, esas entidades con las que nunca se tienen relaciones contractuales precisas" (Da Matta, 2002:102). La calle es el lugar de la desprotección porque allí se puede resultar engañado, robado o confundido con algo que no sé es. En 
saben en dónde conseguir alimentos, ropas, cuáles son los mejores lugares para dormir, etc. Aprendieron a vivir de lo que la calle les ofrece. En este caso, la calle es re-significada como "su" lugar, pues "la calle, en este sentido y a pesar de todo lo que podemos imaginar, es re-significada como un contexto de estabilidad, es decir, como el hogar mismo" (Berho, 2006:25). De este modo se puede afirmar que:

el espacio público no es neutral, es un escenario de conflictos y negociaciones sociales a diversas escalas que lo redefinen continuamente y que, si bien el Estado es el garante para su uso, los grupos sociales concretos establecen estrategias específicas para su utilización, su organización y su significación, desplegando y recreando los referentes identitarios necesarios para su reproducción (Portal, 2009:63).

En definitiva, entendemos que para acercarse a esta problemática resulta adecuada la categoría de Habitantes de la calle dado que da cuenta del universo de personas que conforman esta población, su vínculo con el entorno y las relaciones sociales que entablan. Esta categoría remite tanto a las personas que habitan en la calle estableciendo un vínculo más cercano con el entorno, como a los sujetos que entablan redes dentro del "subgrupo de la calle" en las denominadas "ranchadas" 5 y a las personas que principalmente entablan vínculos con los servicios sociales. Estos diferentes subgrupos identificados reflejan que en el habitar la calle existen diferentes formas de moverse, de estar o de ser.

\section{LA CIUDAD COMO OPORTUNIDAD}

cambio, la casa, su contracara, es concebida como el lugar de la intimidad, de la privacidad, del descanso y de la protección, es el lugar de la familia. En definitiva, el autor afirma que tanto la calle como la casa poseen fuertes connotaciones pues no son sólo espacios geográficos diferenciados sino que son entidades morales y dominios culturales. Para el autor existe una clara oposición entre estos dos espacios: la casa remite al universo de lo controlado, de la armonía y la calma sumada a la idea de calor y afecto. Mientras que la calle hace referencia al mundo del descontrol, alude al movimiento y a la masificación. La casa y la calle demarcan papeles sociales e ideologías diferentes, por lo tanto, a cada esfera de significación corresponden valores y acciones específicas que separan contextos y configuran actitudes.

${ }^{5}$ En la Argentina se habla de "ranchada" para definir a este grupo de habitantes de la calle que comparten su vida cotidiana en la calle. Se entiende que son miembros de un mismo grupo o ranchada, cada una se ubica en un lugar específico y comparten ciertos hábitos y códigos muy vinculados al "mundo de la calle".

Revista de Direito da Cidade, vol.07, no 02. ISSN 2317-7721 pp.518-536 
Desde diversos enfoques se afirma que la experiencia de habitar en la calle es una problemática predominantemente urbana ${ }^{6}$. En la ciudad de Buenos Aires se observa esta tendencia pues allí es donde se halla la mayor cantidad de habitantes de la calle. Esto probablemente se relaciona con que en esta ciudad se ubican las más variadas $y$, a veces, las únicas opciones tanto estatales como privadas para asistirlos ${ }^{7}$. En ella se localizan los establecimientos de la mayoría de las organizaciones sociales, de los programas estatales, las oficinas para trámites relacionados con los subsidios, jubilaciones, etc. También, es en la ciudad donde existe la posibilidad de llevar a cabo diferentes actividades que otorguen un dinero (venta de artículos en la vía pública, changas ${ }^{8}$ relacionadas con algún oficio, mendicidad, limpieza de vidrios de autos, juntar cartones para vender, etc.). Por estas razones es que en la ciudad de Buenos Aires se concentra la mayor cantidad de habitantes de la calle que provienen de la propia ciudad, del conurbano bonaerense, de diferentes provincias del país $y$, en menor medida, de países limítrofes (Rosa, 2012a y 2012b).

Hay que destacar que muchos de los motivos para privilegiar y "sostener" el habitar en la ciudad presentados para los habitantes de la calle son compartidos por otros grupos que viven en situaciones habitacionales vulnerables (inquilinatos, hoteles-pensión, villas miseria, casas ocupadas, etc.). En definitiva:

la ciudad expresa la posibilidad de acceder a oportunidades de trabajo, a equipamientos de educación, salud y esparcimiento más especializados; dicho de otro modo, a un conjunto de atributos complejos e indivisibles que no pueden adquirirse como mercancías. (Catenazzi y Reese, 2010:74).

El habitar la ciudad es central en la cotidianeidad de los sectores vulnerables dado que la ciudad brinda posibilidades y facilidades:

la localización y la permanencia en la ciudad central constituyen un activo priorizado por los sectores populares, en la medida en que les permite mejores accesos a servicios

\footnotetext{
${ }^{6}$ La situación que atraviesan miles de personas y familias en la actualidad al tener que pasar noches y días en las calles es un fenómeno que cada vez es más notorio en las grandes ciudades del mundo.

7 Los servicios destinados para esta población son los hogares de tránsito y los paradores nocturnos. El Gobierno de la Ciudad de Buenos Aires posee los propios y a la vez subsidia otros establecimientos pertenecientes a organizaciones sociales religiosas o no que trabajan con habitantes de la calle.

${ }^{8}$ Término utilizado para hacer referencia a un trabajo de tipo informal, poco calificado y de corta duración.
} 
sociales básicos y a núcleos de empleo, de manera tal de dar respuesta a necesidades básicas" (Herzer, 2008: 184).

Los habitantes de la calle circulan por la ciudad de un servicio a otro, ideando estrategias, coordinando horarios y necesidades durante el día y la noche. Este itinerario entendido como un movimiento constante "se trata de un proceso dinámico que atraviesa etapas a lo largo de la jornada, en las cuales es posible rastrear el trabajo, el descanso y las relaciones interpersonales" (Palleres y Rosa, 2011:278). Este recorrido es realizado diariamente por las calles de la ciudad trasladándose de un servicio a otro sean públicos (hogares de tránsito, paradores, etc.), privados (Entidades religiosas, organizaciones sociales, etc.) o bien concurriendo a algún trabajo, a visitando a un familiar o a un amigo, realizando un tratamiento médico, capacitaciones, etc.

Los habitantes de la calle conocen a qué lugares de la ciudad acudir para obtener lo que necesitan, del mismo modo, saben combinar horarios, direcciones y servicios según sus prioridades del día; por ejemplo, saben que los jueves se pueden bañar en tal organización social, saben además que ese día atiende una trabajadora social que los trata bien y les deja elegir la ropa que más les gusta; luego continúan su recorrido por la ciudad, entran a un locutorio (cibercafé) para ver sus e-mails y más tarde concurren al parador nocturno para cenar y pasar la noche. Otros combinan el "habitar la calle" (sin concurrir para dormir a una institución) con la concurrencia a alguna organización social para recibir comida, ropa, medicamentos o para saber cómo sigue su tramitación de la entrada a un hogar o su jubilación, etc. A partir de este "circuito", trazan por la ciudad recorridos diversos. Uno de los circuitos predeterminados es el que se impulsa desde los programas estatales. Así nos decía una de las coordinadoras de los hogares:

El circuito comienza por los paradores, de paradores va a la admisión, que está en Pavón y Entre Ríos en la oficina 26, y de ahí se deriva a los diferentes lugares. Entra por el parador, después se le abre un legajo único en la DASI, que es donde va a tener una cuestión de requisitos médicos (placa, análisis de orina y de sangre), más que nada para ver que no tenga alguna infecto-contagiosa, sino, después de la cura, ingresa (Entrevista №6).

Esta itinerancia permite comprender la vida cotidiana de los habitantes de la calle y su relación con el entorno. Aprenden a vivir en el circuito de la atención que crean las distintas organizaciones sociales y el Estado. En este entramado es que los habitantes de la calle desarrollan un sin fin de diferentes formas de movilidad y de organización de su tiempo ya que el movimiento 
que realizan por la ciudad es constante, diario y programado. Se puede decir que configuran un mapa específico de la metrópoli según sus prácticas cotidianas. Estas prácticas generan un trazo, una línea que conecta puntos de la ciudad que no es la de los transeúntes típicos. El modo en el cual se constituye el circuito de esta población genera un "mapa" diferente de los recorridos más habituales cuando se piensa la ciudad.

Los habitantes de la calle circulan por la ciudad desplegando habilidades para sostener la vida diaria de un modo casi invisibilizado pero que también forma parte de la experiencia urbana de las grandes ciudades actuales. Por estas experiencias la ciudad es vivida de un modo diferente para esta población a diferencia de otros grupos o sectores que también habitan la ciudad. Se entiende que las experiencias metropolitanas son tanto las:

Prácticas como las representaciones que hacen posible significar y vivir la metrópoli por parte de sujetos diferentes que residen en diferentes tipos de espacio. El concepto de experiencia alude a las muchas circunstancias de la vida cotidiana en la metrópoli y a las diversas relaciones posibles entre los sujetos y los lugares urbanos, a la variedad de usos y significados del espacio por parte de diferentes habitantes. (Duhau y Giglia, 2008:21).

El habitante de la calle usa la ciudad, la recorre, vive en y de ella y construye allí su refugio, su vivienda, su hogar junto con otros y con el entorno (Rosa, 2012a y 2012b).

A continuación se presenta un mapa de la ciudad en el cual se plasmaron las referencias de los lugares típicamente visitados por los habitantes de la calle. Esto se realizó para evidenciar las distancias y recorridos que debe efectuar cotidianamente un habitante de la calle en función de cómo se configuró el campo de atención entre las diversas organizaciones sociales y los servicios estatales. A este itinerario se le suman los recorridos que cada habitante de la calle realiza según sus propias situaciones de vida e intereses. Como se puede observar en los puntos señalados en el mapa muchos de los recorridos implican largas distancias lo que evidencia el movimiento constante al que se ven expuestos para satisfacer sus necesidades diarias. Moverse de un sitio a otro implica tiempo, dinero, el despliegue de estrategias, el uso de medios de transporte, etc.

Mapa Ciudad Autónoma de Buenos Aires, identificación de paradores, hogares y establecimientos de atención a habitantes de la calle 


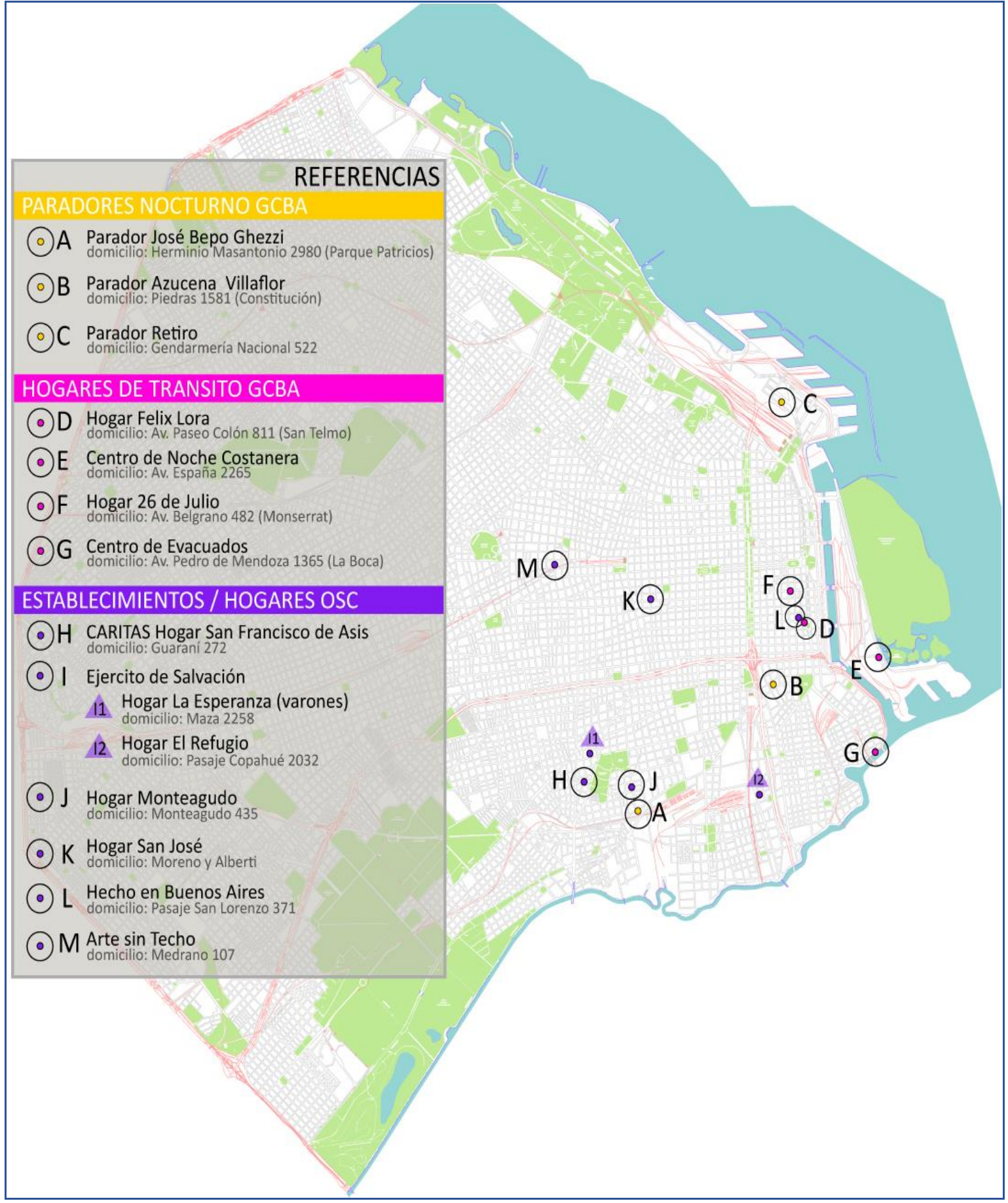

Fuente: Elaboración propia

\section{CONSTRUCCIÓN DE VÍNCULOS}


El itinerario que los habitantes de la calle realizan por la ciudad no solo está relacionado con la meta de conseguir lo necesario para la sobrevivencia sino que también podemos afirmar que la circulación por la ciudad, para un subgrupo ${ }^{9}$ determinado de habitantes de la calle, está signada por la búsqueda de un lugar de pertenencia y para la generación de vínculos sociales duraderos y dadores de sentido. Este subgrupo es denominado, en el marco de esta investigación, como "asistidos potenciados" ${ }^{10}$. Estas son personas que habitando en la calle entablan un vínculo muy particular con los servicios tanto públicos como privados destinados para su atención. Concurren a las organizaciones sociales y a los servicios estatales para recibir alguna prestación (alimentos, ropas, higiene, alojamiento, etc.) pero a la vez asisten en búsqueda de "contención". Buscan establecer vínculos sociales cotidianos, ser escuchados, compartir tiempo con un otro, ser esperados y reconocidos por su nombre, etc. En estos lugares entablan relaciones con profesionales, con el personal (voluntarios o no) y/o con los compañeros (se conocen, conversan, se dan información sobre los servicios, entablan amistad, etc.). Este subgrupo se caracteriza por conservar y fomentar vínculos sociales, intentando correrse del aislamiento y mantenerse en interacción social con otros. Una de las particularidades que posee este sujeto "asistido" es que no

\footnotetext{
${ }^{9}$ Más específicamente, podemos señalar tomando a Grigsby et al (1990) que existen dos tipos de subgrupos además del "asistido potenciado". Uno de ellos es identificado como más cercano a la "subcultura marginal". Dentro de este subgrupo la vinculación se da entre personas que atraviesan la misma situación creando la "subcultura de la calle". Aquí se da un proceso de resocialización a partir de la internalización de ciertos códigos y reglas de la vida en la calle. Los miembros de este grupo, generalmente, consideran que el "habitar en la calle" forma parte de una elección personal y manifiestan que no quieren salir de la misma. Tampoco asisten a servicios de alojamiento aunque algunas veces concurren a algún servicio como puede ser el de duchas o el de ropería. Otro de los subgrupos encontrados por los autores son los que viviendo en la calle no desarrollan vínculos sociales sino que su vida es solitaria por la continua pérdida de apoyos sociales, de este modo llegan a vivir aislados y con un gran deterioro de la salud física y mental.

${ }^{10}$ La categoría de "asistido" fue inspirada a partir de los análisis realizados por Robert Castel (2006). Para este autor, el "asistido" es el que "vive de lo social". Esta es una experiencia que no equivale al total aislamiento sino que conduce a tener otro tipo de relaciones, por ejemplo, con los servicios sociales y con otras personas que atraviesan la misma situación. La categoría de asistido potenciado, creada en el marco de esta investigación, si bien se inspira en la categoría de Robert Castel intenta ahondar en las particularidades que posee para el caso de los habitantes de la calle, por ello no se puede pensar en este grupo como meros "asistidos" sino que lo observado en este campo remite a un potenciamiento en la configuración del "asistido".
} 
quiere ser confundido con el subgrupo de la calle, por el contrario, busca separarse, esto se puede observar en su aspecto físico ya que se encuentran muy limpios y con ropa prolija.

Este subgrupo se inserta en el campo de atención como medio para seguir vinculados con el entorno social, y así entran en un entramado conformado por organizaciones sociales y por programas estatales. Estas personas que se insertan en este itinerario adquieren nuevas formas de llevar a adelante su vida cotidiana. Este es un esquema muy complejo pues deben considerar los horarios de ingreso y egreso, conocer los recorridos más rápidos y baratos para llegar, saber qué consiguen en cada lugar o con quién tienen que hablar, etc., es decir, que requieren de muchas habilidades. De esta manera, se debe "aprender" a ser miembro de este subgrupo dado que se necesitan destrezas específicas que solo se asimilan en la práctica y de la mano de "expertos" como puede ser alguien que hace más tiempo que vive en esta situación. Hay un proceso de formación como "asistido" ya que se deben conocer las estrategias que se deben desplegar, cuáles son las apariencias, las fachadas y los modales necesarios en cada medio (Goffman, 2006). Se desarrollan adaptaciones de la persona en la institución para recibir asistencia, de este modo, intentan transmitir ciertas impresiones a través del lenguaje, el vestuario, la apariencia, etc. Buscan desarrollar un tipo de impresión que saben deben proyectar, por ejemplo, a los agentes estatales. Adquirieron el conocimiento que en estas instituciones buscan identificar a los "pobres verdaderos" (por ello desde el Estado en el caso de los habitantes de la calle se extiende un "certificado de situación de calle", este certifica que la persona hace más de un año que se encuentra habitando en la calle) y excluir a los "aprovechadores". Si bien Goffman sostiene que este tipo de adaptaciones son comunes en, por ejemplo, los beneficiarios de planes sociales; en el caso de los "asistidos potenciados" pudimos observar que este tipo de impresiones que se buscan dar llegan a un punto límite dado que esta impresión les debe permitir encontrar un lugar de pertenencia. En este sentido es que van adaptando sus subjetividades y acciones para ser aceptados en los servicios sociales. Intentan ser reconocidos tanto por los miembros de las organizaciones sociales como por los agentes estatales, buscan entablar vínculos estrechos con ellos. Por ello sus "verdaderas" intensiones no se pueden asociar con la de otros beneficiarios de planes sociales que despliegan variedad de "actuaciones" para lograr un subsidio, un plan, etc.; los 
"asistidos potenciados" van más allá, no buscan conseguir "algo" e irse, sino que lo que buscan es quedarse, ser parte.

Se puede decir que los miembros de este subgrupo son personas que tienen una vida de relación pero desde un rol degradado, es decir, que no entablan un vínculo profundo con quienes se relacionan sino que el lazo que entablan es precario y frágil. Al entrar en el circuito los habitantes de la calle se consolidan como sujetos dependientes del campo de atención dado que su día -y noche- se conforma a partir de la construcción de un itinerario que combina los diversos sitios y recursos que se ofrecen para asistirlos. De este modo, este campo de atención refuerza su itinerancia por la ciudad, sin plantear alternativas superadoras de su presente de inestabilidad e incertidumbre. Sin embargo, no se puede negar la relevancia que tienen estos vínculos cuando se atraviesa esta situación tan vulnerable. Estos vínculos frágiles se tornan centrales, principalmente, porque son los únicos. Es por esto que la "pertenencia" el campo de atención es un "componente central de sus estrategias de supervivencia física, social e incluso simbólico" (Cabrera, 1998:380-1).

De este modo, este subgrupo rompe el clásico estereotipo del solitario que pareciese como si estuviera inmerso en "otro mundo" o inserto en el "submundo marginal". Por el contrario, su objetivo es formar parte de un grupo para entablar relaciones duraderas y dadoras de sentido para su propia existencia. Como podemos observar, el recorrido por la ciudad que realiza este subgrupo se relaciona más significativamente a un modo de vida de tipo tradicional comunitario que al concebido como típicamente urbano.

\section{REFLEXIONES FINALES}

La experiencia urbana de los habitantes de la calle evidencia "capas superpuestas" de entramados urbanos. Es decir, capas de ciudades (aparentando ser una sola ciudad) que conviven dependiendo de las prácticas y recorridos que allí se realizan. Las prácticas urbanas generalmente están asociadas con la circulación, el entretenimiento y el anonimato. Sin embargo, estas se superponen con las vivencias -poco exploradas desde el punto de vista urbano- de los habitantes de la calle que se apropian de este entorno para reproducirse, crear relaciones sociales, trabajar, etc. Esta "capa" tan significativa para muchos parece inexistente cuando se planifican las ciudades pues este habitante no es concebido como un habitante de la ciudad. 
Los habitantes de la calle son una población, compuesta por diversidad de subgrupos, que comparten tener poca visibilidad social. Sus recorridos, experiencias y movimientos por la ciudad son prácticamente invisibles. Sin embargo, todos los días en la mayoría de los barrios de la ciudad hay miles de personas y familias que circulan de un lugar a otros intentando reproducir su vida y lograr un lugar de pertenencia a través del desarrollo de un cierto tipo de relaciones sociales.

Este modo de vida sería imposible de lograr en otro entorno. La ciudad, con sus particularidades, y el circuito de atención para esta población son los que permiten la conformación del subgrupo de asistidos potenciados. Su itinerancia es necesaria para conseguir lo necesario para atravesar el día a día. En este sentido, la ciudad facilita la emergencia de estos grupos y sus modos de vida permiten el desarrollo de experiencias urbanas diversas que pueden convivir independientemente unas de otras.

El modo de vida de los habitantes de calle pone en cuestión los supuestos dados al espacio público y las interacciones que allí se desarrollan, pues con sus prácticas cotidianas y sus apropiaciones, tensionan las premisas que sostienen que en el espacio público todo es anonimato, individualismo, instrumentalidad y relaciones sociales precarias. Ellos evidencian que ese espacio compartido puede tener múltiples significados dependiendo de los sentidos que se le otorguen. La ciudad entonces es un escenario que posibilita observar este tipo de relaciones. De este modo, las relaciones sociales pueden ser pensadas como relaciones espaciales dado que se inscriben en un territorio determinado y generan diversas construcciones de lo espacial.

Este caso deja en evidencia que es una tarea difícil e innecesaria definir la ciudad como un todo, como un espacio sin grietas ni ambigüedades. Por ello, como sostiene Rizo (2012), la ciudad:

Se convierte en un objeto de estudio difícil de abordar de forma completa, cerrada. Los afanes de comprensiones e interpretaciones totalizadoras se convierten en intentos realizados en vano, ya que se distancian en gran medida de la lógica incierta del mundo urbano (2012:74).

En este sentido, es que la experiencia de los habitantes de la calle permite realizar un acercamiento diferente a los estudios de lo urbano pues resulta un caso interesante para entender cómo se da la construcción de la ciudad y cuáles son los modos posibles de ser habitante de la ciudad. En resumen, entendemos que el habitar en estos espacios compartidos nos dice mucho de la propia ciudad y de los procesos que en ella se dan. 


\section{REFERENCIAS BIBLIOGRÁFICAS}

BERHÓ, M.; "Identidad marginal entre personas sin hogar de la ciudad de Temuco". Chile, Revista CUHSO 11 (1), Centro de Estudios Socioculturales de la UC Temuco, 2006.

CABRERA, Pedro; Huéspedes del Aire. Sociología de las personas sin hogar en Madrid. Madrid, UPCO, 1998.

CATENAZZI, A y REESE, E.; Derecho a la ciudad. La dinámica de crecimiento urbano, el déficit habitacional y las asignaturas pendientes. Revista Voces del Fénix, 1, 2010.

DUHAU, Emilio y GIGLIA, Ángela; Las reglas del desorden: habitar la metrópoli. (México: Siglo XXI Editores) Universidad Autónoma Metropolitana, Unidad Azcapotzalco, 2008.

DA MATTA, Roberto; A Casa \& A Rua, Río de Janeiro, Rocco, 1997.

; Carnavales, malandros y héroes. Hacia una sociología del dilema brasileño, México, Fondo de Cultura Económica, 2002.

DE CERTEAU, Michel; La invención de lo cotidiano I. Artes de hacer, Méjico, Universidad Iberoamericana. 2000

DE LA PEÑA GABRIELA; "Simmel y la escuela de chicago en torno a los espacios públicos en la ciudad". En: Valladares Vielman Luis Rafael (Compilador) La Ciudad. Antecedentes y nuevas perspectivas. Guatemala: CEUR, USAC, 2012.

GUILLAMÓN, Marrero, Isaac; "La producción del espacio público Fundamentos teóricos y metodológicos para una etnografía de lo urbano". (Con) textos, Revista d'antropologia i investigació social. Número 1. Maig de 2008. Páginas 74-90, 2008.

GOFFMAN, Erving; Estigma. La identidad deteriorada. Buenos Aires, Amorrurtu, 2006.

GRIGSBY, C.; BAUMANN, D.; GREGORICH, S.; Roberts, C.; Desaffiliation to Entrenchment: A model for Understanding Homelessness Journal of Social Issues 46, pp. 141 a 156, 1990.

HERZER, Hilda (Comp.) Con el corazón mirando al sur. Transformaciones en el sur de la ciudad de Buenos Aires. Buenos Aires, Espacio Editorial, 2008.

LINDÓN, Alicia; "La construcción socioespacial de la ciudad: el sujeto cuerpo y el sujeto sentimiento" en Cuerpo, Emociones y Sociedad, Córdoba, №1, Año 1, p. 06-20, Dic., 2009.

LOFLAND, L. H.; The Public Realm. Exploring the City's Quitessential Social Territory. Nueva York: Aldine de Gruyter, 1998. 
MARCÚS, Juliana (2006) "Ser madre en los sectores populares: una aproximación al sentido que las mujeres le otorgan a la maternidad" Revista Argentina de Sociología. Buenos Aires, vol.4 n 07 noviembre-diciembre, 2006.

PARK, Robert Ezra; La ciudad y otros ensayos de ecología urbana. Barcelona: Ediciones del Sebal, 1999.

PALLERES, Griselda y Rosa, Paula; "Reproducción de prácticas y cotidianeidad: Políticas públicas y situación de calle en la Ciudad de Buenos Aires". En Di Virgilio, Mercedes; Herzer, Hilda; Merlinsky, Gabriela y Rodríguez, Carla (Comp.) La cuestión urbana interrogada. Transformaciones urbanas, ambientales y políticas públicas en Argentina. Ediciones El café de las ciudades. Buenos Aires, Argentina. ISBN 978-987-25706-1-3.

ROSA, Paula (2009) "Las prácticas de encierro hoy: Reflexiones de la mano de Michel Foucault sobre los programas destinados a personas en situación de calle" Revista Afuera - Estudios De Crítica Cultural. Buenos Aires, n 6 Mayo, 2011.

PORTAL, María Ana; "Las creencias en el asfalto. La sacralización como una forma de apropiación del espacio público en la ciudad de México". Cuadernos de Antropología Social № 30, 2009.

RIZO, Marta; Conceptos para pensar lo urbano. el abordaje de la ciudad desde la identidad, el habitus y las representaciones sociales. En: Valladares Vielman Luis Rafael (Compilador) La Ciudad. Antecedentes y nuevas perspectivas. Guatemala: CEUR, USAC, 2012.

Rosa, Paula; "Pobreza urbana y desigualdad: La asistencia habitacional a las personas en situación de calle en la Ciudad de Buenos Aires". En: Bolívar, Teolinda y Erazo Espinoza, Jaime; Hábitat Popular e Inclusión Social, Ciudad de Quito-Ecuador, CLACSO. ISBN: 9789978673287, 2012a. Disponible en: http://bibliotecavirtual.clacso.org.ar/clacso/gt/20120409043609/gthi2-15.pdf

"Ser" habitante de la Ciudad Autónoma de Buenos Aires: Experiencias y procesos de una ciudad en transformación. Boletín Científico Sapiens Research, Colombia. Volumen 2 número 1, páginas 60-65. ISSN-e: 2215-9312, 2012b. Disponible en: http://www.sapiensresearch.org/elboletin-cientifico-sapiens-esearch/67?open=anything,

TORTEROLA, Emiliano; Lazo social y metrópolis. La comunidad en los orígenes de la sociología urbana: Gerg Simmel y Robert E. Park. En: De Marinis, Pablo (2012); Comunidad: estudios de teoría sociológica. Buenos Aires. Prometeo, 2012.

SAUCEDO, I.A. y TARACENA, B. E.; "Habitar la calle": pasos hacia una ciudadanía a partir de este espacio. Revista Latinoamericana de Ciencias Sociales, Niñez y Juventud, 1 (9) 269-285, 2011.

SIMMEL, Georg; El individuo y la libertad. Ensayos de crítica de la cultura. Barcelona: Península, Serie Historia, Ciencia, Sociedad, 1986. 
VIOTTI, Nicolás; "Una sociología de la deriva”. Revista Apuntes de Investigación del CECYP, No 13, 2008. Disponible en:http://www.apuntescecyp.com.ar/index.php/apuntes/article/view/32

Trabalho enviado em 08 de março de 2015.

Aceito em 17 de março de 2015. 